\title{
Best practice in therapeutic drug monitoring
}

\author{
Annette S. Gross \\ Department of Clinical Pharmacology, Royal North Shore Hospital, St Leonards NSW 2065, Australia
}

\begin{abstract}
It is the goal of Therapeutic Drug Monitoring (TDM) to use drug concentrations to manage a patient's medication regimen and optimise outcome. Limited resources require that drug assays should only be performed when they do contribute to patient management. For this to be the case a therapeutic drug monitoring service has a far greater role than just therapeutic drug measuring. This article describes the roles and functions of a Best Practice TDM service. The features which can and should be strived for in each step of the TDM process - the decision to request a drug level, the biological sample, the request, laboratory measurement, communication of results by the laboratory, clinical interpretation and therapeutic management-are discussed.
\end{abstract}

Keywords: therapeutic drug monitoring, therapeutic management, clinical interpretation

\section{Introduction}

Within the health care system there are continuing pressures to provide services at the lowest possible cost. The role of many drug assay laboratories is to measure the concentration of a therapeutic drug in a blood sample and relate this number to a therapeutic range published in the literature. For this they receive regular funding or a standard fee. However therapeutic drug measuring is only one part of therapeutic drug monitoring (TDM) which provides expert clinical interpretation as well as the concentration. There is rarely financial provision for this. Nevertheless expert interpretation of a drug concentration measurement is essential to ensure full clinical benefit. Only clinically meaningful tests should be performed and limited funds should not be wasted on measurements which cannot be interpreted and do not assist patient management.

Clinicians routinely monitor drug pharmacodynamics by directly measuring physiological indices of therapeutic response e.g. lipid concentrations, blood glucose, blood pressure, clotting tests. For many drugs there is either no readily available measure of effect or it is insufficiently sensitive. Large interindividual variation in the relationship between dose and response can make individualising drug dosage difficult, for example drugs with narrow therapeutic indices, large interindividual variation in pharmacokinetics, or concentration-dependent pharmacokinetics. In other cases it is difficult to distinguish between the

progress of the disease and the pharmacological effects of a drug. It is in these situations that TDM is an essential part of clinical management.

The process of TDM is predicated by the assumption that there is a definable relationship between dose and plasma or blood drug concentration and between the latter and pharmacodynamic effects (Figure 1). The contribution of pharmacokinetic variability to differences in dose requirements can be identified by measuring the concentration of the drug at steady-state and modifying the dose to attain a desired drug concentration known to be associated with efficacy and not toxicity. However there is substantial interindividual pharmacodynamic variability at a given plasma concentration [1] and hence a range of concentrations rather than a single level is usually targeted. For a limited number of drugs where there is a better relationship between plasma or blood concentration and response than between dose and response, the measurement of plasma or blood concentrations has become a valuable surrogate index of drug exposure. It is the goal of TDM to optimise a patient's clinical outcome by managing their medication regimen with the assistance of measured drug concentrations.

Therapeutic drug monitoring is a multidisciplinary function. Accurate and clinically meaningful drug concentrations can only be obtained by collaboration between scientists, clinicians, nurses and pharmacists (the TDM team) and excellent communication is necessary to ensure that best practice in TDM is achieved. To date therapeutic drug monitoring services have usually been set up within large hospitals and this article focuses on monitoring of hospital inpatients and outpatients. However patients in 
Pharmacokinetic

variability
Pharmacodynamic

variability

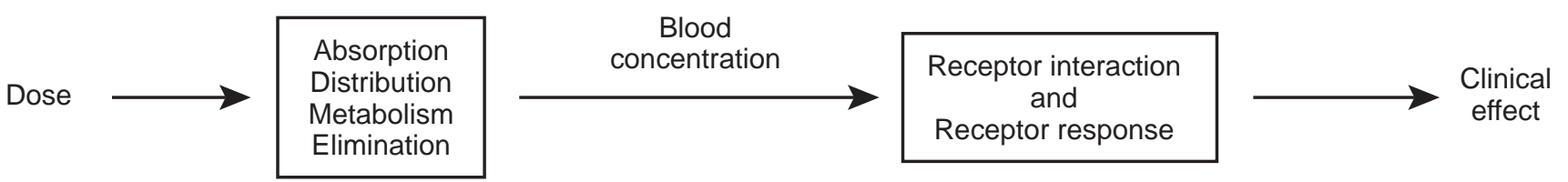

Figure 1 Pharmacokinetics and pharmacodynamics contribute to variability in the relationship between drug dose and response.

the wider community are managed by their doctors utilising pathology laboratories, often without clinical interpretation of the drug concentration measured [2]. This arises partly because the 'value adding' provided by clinical interpretation is not directly funded by any health care system model. However, the principles of best practice TDM could be readily applied to the community setting and should be increasingly easy to incorporate as information technology in general practice continues to improve.

Many excellent articles describing TDM principles and the limitations of therapeutic ranges have been published [3-5]. Only a limited number of articles have been published which demonstrate the cost-effectiveness of therapeutic drug monitoring, and there continues to be debate as to whether these services make a cost-effective contribution to patient care [6-10]. However clinicians continue to order drug concentrations. In 1995 a document entitled Best Practice in Therapeutic Drug Monitoring was published in Australia [11], endorsed by the Australasian Society for Clinical and Experimental Pharmacologists and Toxicologists (ASCEPT); its aim was to describe an 'ideal' TDM service. This article builds on that document and identifies those features of a Best Practice TDM service which can and should be attained.

\section{The TDM process}

\section{Decision to request a drug level}

Drug concentration measurements are requested to assist the manipulation of a patient's current medication regimen (TDM) or to screen for a medicine or substance which may be contributing to the presentation of a medical emergency (clinical toxicology). The goals for the provision of comprehensive clinical toxicology service differ from those of a TDM service and will not be considered here.

The indications for TDM are listed in Table 1. In an ideal environment drug levels would only be requested when there is an appropriate indication. In a large hospital with a high staff turnover, continuing education by the members of the TDM team about the criteria for rational drug level requests is required [12]. Procedures may also be implemented to assess whether requests for drug assays are warranted before
Table 1 Reasons for requesting drug concentration measurements.

Toxicity suspected-toxic concentrations?

Lack of response - subtherapeutic concentrations?

Assessment of compliance with medication regimen

Assess therapy following a change in dosage regimen

Change in clinical state of the patient

Potential drug interaction due to change in comedications

Manifestations of toxicity and disease state are similar

the assays are actually performed, thereby ensuring the rational utilisation of resources. This is often time consuming for senior personnel but can be cost-effective as expensive tests which do not assist either immediate or long-term patient management are not performed.

\section{The biological sample}

Once the decision to monitor the concentration of a therapeutic drug has been made, it is important that a biological sample is collected which will provide a clinically meaningful measurement.

Blood samples should be collected once the drug concentrations have attained steady-state i.e. at least 5 half-lives at the current dosage regimen. Levels approximating steady-state may be reached earlier if a loading dose has been administered. However drugs with long half-lives should be monitored before steady-state is achieved to ensure that individuals with impaired metabolism or renal excretion are not at risk of developing toxicity at the initial dosage regimen prescribed e.g. amiodarone, perhexiline. If toxicity is suspected then concentrations should be monitored as soon as possible. Likewise, immediate assay might be indicated if there is poor therapeutic control, as in rapid atrial fibrillation, when loading doses could be useful. To interpret the result, details of the dosage regimen (dose and duration) are essential.

Blood or plasma concentrations change throughout a dosage interval and the time of the blood sample relative to the time of administration of the dose must be known to enable sensible interpretation. Absorption is variable after oral administration and blood samples should be 
Table 2 The information required to interpret a drug concentration.

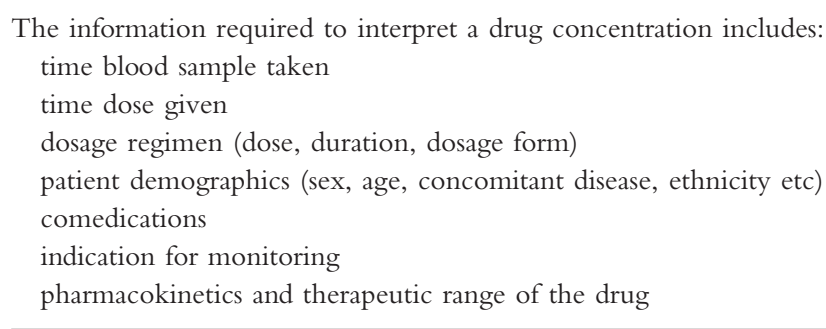

collected in the elimination rather than in the absorption or distribution phases. Usually blood samples are collected at the end of the dosage interval (trough). For antibiotics given intravenously, 'peak' concentrations $(30 \mathrm{~min}$ after cessation of the infusion) are also measured. For aminoglycosides given by bolus injection, samples should be taken at least $1 \mathrm{~h}$ post dose to avoid the distribution phase. Concentrations measured at these time points can be related to published therapeutic ranges which are usually based on prospective studies relating trough drug concentrations measured at steady-state to pharmacodynamic response. For antibiotics, ranges are based on both peak and trough concentrations. For drugs with short halflives, samples taken at other times during the dosage interval may give results which can be interpreted reliably only if a pharmacokinetic approach is used. However for drugs with long half-lives (e.g. $24 \mathrm{~h}$ or greater) stable levels throughout a dosage interval may allow interpretation of samples taken at earlier post-distribution times e.g. digoxin may be monitored 6 to $8 \mathrm{~h}$ after an oral dose.

Usually medicines are monitored in venous blood, serum or plasma and it is very important that the appropriate matrix is assayed. In general plasma or serum concentrations are comparable but the blood collecting tube used can be important. For example lithium heparin is an inappropriate anticoagulant for lithium samples and certain gel separator tubes should be avoided for some drugs such as phenytoin [13]. Whole blood must be sampled for some drugs e.g. for cyclosporin A, which distributes between erythrocytes and plasma in a temperature dependent manner (whole blood concentrations are measured) in order to obtain a reliable and artefact-free result. In small babies capillary blood samples are collected for TDM but despite extensive research examining the utility of saliva measurements, other biological fluids are not routinely sampled [14].

\section{The request}

The information needed to interpret a drug concentration result is given in Table 2. Patient demographics are critically important so that the contribution of age, disease state, ethnic group etc. to interindividual variation in pharmacokinetics and pharmacodynamics can be considered. These details must be effectively communicated to the members of the TDM team with a drug assay request. As the ordering of pathology requests becomes increasingly computerised, and interfaced with clinical databases, the collection of patient demographics and information on dosage regimens and comedications should become more reliable and accurate. It will always be essential to train the staff who collect blood samples to record the time at which the blood sample is taken and also to establish and record not only the time at which the last dose was administered but also the full dosage history.

When a drug which is commonly measured for TDM is suspected of causing toxicity, it is very important for requesting clinicians to clearly communicate the expectation of a high concentration and the need for a rapid feedback of results.

\section{Laboratory measurement}

Ideally a quality drug assay should be performed within a clinically useful time frame. In large chemical pathology laboratories staffed by highly skilled scientists and equipped with state-of-the art automated analysers, many clinicians assume that the results obtained will be accurate. Certain procedures are necessary for this to be the case.

Most importantly the laboratory should be accredited to perform drug analyses by the relevant regulatory authorities e.g. the National Association of Testing Authorities in Australia. This accreditation will give assurance that appropriate techniques are being used by suitably trained personnel. However an infrequent and brief laboratory inspection may not always ensure that optimal procedures are employed for all assays at all times.

The analytical laboratories should ensure that:

- Procedures are in place to obtain any information which is missing from the drug assay request and needed for appropriate clinical interpretation of the result (e.g. dosage regimen, time of blood sampling).

- The accuracy, precision, sensitivity and specificity of each assay is documented and assessed regularly.

- Wherever possible assay performance is evaluated using an external quality assurance program, which provides a rapid turn around of results, comprehensive feedback on performance and has a large number of subscribers e.g. Heathcontrol Therapeutic Drugs Scheme or College of American Pathologists. However some assays are not included in external assurance schemes. For these compounds internal quality controls should be prepared by personnel, other than those performing the 
assays, from separate weighings of reference standards. To evaluate the performance of these assays, biological samples should be exchanged with other laboratories known to be measuring these drugs.

- Each analytical run is performed with the appropriate number of calibration standards and quality controls.

- Senior laboratory staff verify the assay results in light of the clinical request.

- The result should be available for clinical interpretation within a clinically meaningful time interval. In the ideal therapeutic drug monitoring service the laboratory turn-around-time for all assays should be so rapid, that the drug concentration would be available to the clinician before the patient receives the next dose. For many drugs the analytical techniques used, and their associated costs, dictate that assays are performed in batches at predetermined times and the drug concentrations may consequently not be available for a number of dosage intervals.

Rapid and cost-effective measurement of most drugs for which TDM is indicated can be achieved using commercial kits run on automated analysers using a number of different methodologies including fluorescence polarisation immunoassay. However kits are not available for all drugs for which therapeutic monitoring is indicated (including amiodarone, sotalol and perhexiline). Chromatographic techniques are usually used to measure these drugs. Furthermore for a small number of indications, such as the management of pregnant epileptics, therapy can best be controlled by measuring the free or unbound, rather than the total, drug concentration in plasma. For therapeutic drug monitoring free drug levels are usually measured by ultrafiltration [15]. Chromatographic and ultrafiltration techniques are time consuming and require highly trained staff. It is most cost-effective for these assays to be performed in only a limited number of centres of excellence with appropriately qualified scientists and stringent quality assurance.

It is important to use quality control material from an independent source whenever possible and to be aware of any limitations of the commercial kits being used. When necessary clinicians should be advised about interpretation of complex results e.g. interference from endogenous digoxin-like immunoreactive factors in patients with renal failure or neonates, potential contribution of cyclosporin metabolites to the cyclosporin concentration reported etc.

Reliable laboratory information systems can support a comprehensive therapeutic drug monitoring service in a number of ways. By minimising manual data transfer and the possibility of transcription errors, repetitive manual data checking can be reduced and the reliability of the data generated will be improved. Importantly an ideal laboratory information system should readily and rapidly provide statistics on many aspects of the TDM service facilitating appropriate audit of the drug assays and their associated costs. This database could also be a rich source of information on drug concentrations, dosage schedules and patient demographics ideally suited for hypothesis generating research [16].

\section{Communication of results by the laboratory}

The laboratory should ensure that the drug concentration is available for clinical interpretation as quickly as possible after it has been verified by senior staff. For appropriate interpretation the laboratory report should be designed to incorporate all the details listed in Table 2. It should also identify clearly the analytical technique used if there are significant clinical implications. For example the contribution of cyclosporin A metabolites to the concentration measured depends on which commercial antibody has been used. Assays for the same patient performed in different laboratories using different antibodies can vary in the contribution of the metabolite to the concentration measured and this can be clinically significant [17].

The drug concentrations measured are reported in either mass or molar units. To relate concentration back to dose, mass units are preferable. However in many instances assays are performed in biochemistry laboratories and molar units are used. In order to interpret the drug concentration obtained in an individual patient, the laboratory should be able to readily convert the drug concentration measured between molar and mass units.

The drug assay results should also clearly state the therapeutic concentration range for the drug assayed. The therapeutic ranges commonly cited have been established during prospective studies of clinical outcome in relatively small numbers of patients and the ranges quoted often differ between laboratories. This difference can arise because local clinicians have strong views on the appropriate therapeutic range for a given drug or because the therapeutic ranges reported are based on different published studies. Critical assessment of the original literature and moves toward consensus recommendations for therapeutic ranges are to be encouraged. It must be remembered that different indications for therapy as well as age or ethnic differences in pharmacodynamics could result in different therapeutic ranges being appropriate for different patient populations.

\section{Clinical interpretation}

Clinical interpretation can 'add value' and transform a therapeutic drug measuring service into a TDM service. The importance of the clinical interpretation of the drug concentration measured and its contribution to individualisation of a drug dosage regimen cannot be over emphasised. Just relating a drug concentration to a 
published therapeutic range is not an adequate interpretation e.g. a digoxin concentration must be interpreted in light of the creatinine and potassium concentrations, the presence of acidosis or the administration of interacting drugs as well as the patient's clinical state.

The concentration measured must always be interpreted in light of the clinical response, the demographics and clinical status of the individual patient, the dosage regimen used, the indication for therapeutic drug monitoring and the pharmacokinetic characteristics of the drug. Ideally interpretation and advice should be provided with every report by suitably qualified and experienced staff. This should be distributed to the requesting clinician as rapidly as possible, electronically if desired. Prompt alerting of drug concentrations outside the expected therapeutic range will assist in rapid intervention in patients at risk of toxicity.

The ideal TDM team should consist of clinical pharmacologists, clinical pharmacists and analytical scientists. Members of the TDM team with appropriate clinical expertise should be available for consultation regarding individual patients and be prepared to liaise between the medical staff managing the patient, the laboratory staff measuring the drug concentration and the pharmacists and nurses handling the patient's medications. Most institutions do not have the luxury of a TDM team but will have some of the individuals necessary to provide an interpretative service.

Dosage prediction techniques using a number of software packages have been used to help individualise drug dosage regimens. Bayesian techniques have been successfully used to estimate the pharmacokinetic parameters of a drug in a given patient [18] and hence used to predict appropriate dosage regimens to attain defined target concentrations [19]. Pharmacokinetic interpretive services improve patient care [20, 21]; however to date their use has been restricted to large hospitals. The dosage predictions, often including a graph, are ideally suited for incorporation into the report form issued by a TDM service and can be performed by interested clinical scientists, pharmacists or pharmacologists. In addition individualised dosage prediction using Bayesian techniques is a very useful tool for teaching clinical pharmacokinetic concepts.

\section{Therapeutic management}

The clinician caring for a patient will modify a drug dosage regimen in light of all available information. If the members of the TDM team are well respected, many physicians will accept and implement their recommendations for dosage adjustment, and seek their further advice.

\section{Other core functions of a TDM service}

\section{Education}

The staff of a comprehensive TDM service should actively promote an understanding of the principles of rational drug concentration measurement and interpretation. Formal teaching of the basic principles of TDM should be included in the initial training of doctors, pharmacists and nurses. Continuing education of these professionals in hospitals and the general community is also required. Current trends towards greater consumer involvement in their management suggests the need for even wider educational programmes.

\section{Research and development}

Ongoing research and development have pivotally important roles to play in a comprehensive TDM service. TDM professionals should perform research into the mechanisms or causes responsible for clinical problems highlighted by the monitoring of drug concentrations e.g. drug interactions. They should also actively target and investigate areas where TDM could improve drug therapy. Research into TDM issues should be encouraged including prospective studies of pharmacoeconomic outcomes and investigation of the concentration-effect relationships of new and established chemical entities [22, 23]. Senior staff in TDM services should be available to supervise postgraduate students involved in TDM related research and to present these results at conferences and in the literature. On a regular basis all aspects of the TDM service and its utilisation by local clinicians should be audited and the needs of the customers assessed and addressed.

\section{The bigger picture}

Staff with appropriate qualifications should be encouraged to participate actively in TDM services. These individuals will require comprehensive training and continuing education in order to satisfy minimum standards of competency. They should be encouraged to become involved in professional societies with an interest in TDM: such as the International Association for Therapeutic Drug Monitoring and Clinical Toxicology.

Issues to be addressed in the future include how to fund TDM services, the need for expert clinical interpretation of drug concentration measurements performed in general practice and how clinical interpretation will be provided as technology allows drug concentration measurements to move from the pathology laboratory to the bedside.

The ideas and hard work of Susan Tett, John Ray and Ray Morris, who are the principal authors of the document Best Practice in Therapeutic Drug Monitoring, are wholeheartedly acknowledged. 


\section{References}

1 Levy G, Ebling WF, Forrest A. Concentration- or effectcontrolled clinical trials with sparse data. Clin Pharmacol Ther 1994; 56: 1-8.

2 Campbell M. Community-based therapeutic drug monitoring. Clin Pharmacokinet 1995; 28: 271-274.

3 Aronson JK, Hardman M. Measuring plasma drug concentrations. Br Med J 1992; 305: 1078-1080.

4 Birkett DJ. Therapeutic drug monitoring. Australian Prescriber 1997; 20: 9-11.

5 Spector R, Park GD, Johnson G, et al. Therapeutic drug monitoring. Clin Pharmacol Ther 1988; 43: 345-353.

6 Bussey HI, Hoffman EW. A prospective evaluation of therapeutic drug monitoring. Ther Drug Monit 1983; 5: 245-248.

7 Tonkin AL, Bochner F. Therapeutic drug monitoring and patient outcome. Clin Pharmacokinet 1994; 27: 169-174.

8 Eadie MJ. The role of therapeutic drug monitoring in improving the cost effectiveness of anticonvulsant therapy. Clin Pharmacokinet 1995; 29: 29-35.

9 Eilers R. Therapeutic drug monitoring for the treatment of psychiatric disorders. Clin Pharmacokinet 1995; 29: 442-450.

10 Eadie MJ. Indications for plasma drug monitoring in patients with epilepsy. Pharmacoeconomics 1997; 11: 343-349.

11 Tett S, Ray J, Morris R. Best Practice in Therapeutic Drug Monitoring 1995. Published.

12 Carroll DJ, Austin GE, Stajich GV, et al. Effect of education on the appropriateness of serum drug concentration determination. Ther Drug Monit 1992; 14: 81-84.

13 Quattrochi F, Karnes HT, Robinson JD, et al. Effect of serum separator blood collection tubes on drug concentrations. Ther Drug Monit 1983; 5: 359-362.

14 Pichini S, Alteri I, Zuccaro P, et al. Drug monitoring in nonconventional biological fluids and matrices. Clin Pharmacokinet 1996; 30: 211-228.

15 Wright JD, Boudinot FD, Ujhelyi MR. Measurement and analysis of unbound drug concentrations. Clin Pharmacokinet 1996; 30: 445-462.

16 Gex-Fabry M, Balant-Gorgia AE, Balant LP. Therapeutic drug monitoring databases for postmarketing surveillance of drug-drug interactions: evaluation of a paired approach for psychotropic medication. Ther Drug Monit 1997; 19: 1-10.

17 Morris R. Cyclosporin A monitoring in Australia: Consensus recommendations. Ther Drug Monit 1994; 16: 570-576.

18 El Desoky E, Meinshausen J, Bühl K, et al. Generation of pharmacokinetic data during routine therapeutic drug monitoring: Bayesian approach vs pharmacokinetic studies. Ther Drug Monit 1993; 15: 281-288.

19 Thomson AH, Whiting B. Bayesian parameter estimation and population pharmacokinetics. Clin Pharmacokinet 1992; 22: 447-467.

20 Ried LD, McKenna DA, Horn JR. Meta-analysis of research on the effect of clinical pharmacokinetics services on therapeutic drug monitoring. Am J Hosp Pharm 1989; 46: 945-951.

21 Ried LD, Horn JR, McKenna DA. Therapeutic drug monitoring reduces toxic drug reactions: a meta-analysis. Ther Drug Monit 1990; 12: 72-78.

22 Mattson RH Antiepileptic drug monitoring: a reappraisal. Epilepsia 1995; 36 (Suppl 5): S22-S29.

23 Freeman DJ, Oyewumi LK. Will therapeutic drug monitoring have a place in clozapine therapy? Clin Pharmacokinet 1997; 32: $93-100$. 